\title{
Expression pattern of human SERPINE2 in a variety of human tumors
}

\author{
YING YANG $^{1}, \mathrm{XIANGKE} \mathrm{XIN}^{1}, \mathrm{XING} \mathrm{FU}^{1}$ and DANMEI XU $\mathrm{X}^{1,2}$ \\ ${ }^{1}$ Department of Haematology, Tongji Hospital, Tongji Medical College, \\ Huazhong University of Science and Technology, Wuhan, Hubei 430030, P.R. China; ${ }^{2}$ Department of Haematology, \\ Imperial College London, Hammersmith Hospital, London, W12 0HS, UK
}

Received March 12, 2017; Accepted December 21, 2017

DOI: $10.3892 / \mathrm{ol} .2018 .7819$

\begin{abstract}
Serine proteinase inhibitor, clade E member 2 (SERPINE2), also known as protease nexin-1 (PN-1), is a member of the serpin family. Despite several reported roles of SERPINE2 in tumor development the histological distribution of SERPINE2 and its expression levels in a large variety of tumors remains unclear. Through expressed sequence tag database analysis, immunohistochemical staining of tissue microarrays and a literature review, it was revealed that SERPINE2 expression varied according to growth stages and tissue types. SERPINE2 is differentially expressed in a number of tumors and their normal tissue counterparts. SERPINE2 is identified most abundantly in adenocarcinomas. SERPINE2 serves diverse roles in a variety of tumors and therefore may serve as a promising biomarker for tumor diagnosis and prognosis.
\end{abstract}

\section{Introduction}

SERPINE2, a 43-kDa protein first found as a neurite-promoting factor in the culture medium of glial cells (1), was subsequently proven as a member of the SERPIN family that has serine protease inhibitory activity $(2,3)$. It also belongs to the nexin protease family according to its amino acid sequence, therefore also commonly called protease nexin-1 (PN-1) (4). SERPINE2 mainly presents in the extracellular matrix (ECM) and is secreted by many cell types, including endothelial cells, fibroblasts, macrophages, platelets, smooth muscle cells, chondrocytes, astrocytes and tumor cells (5-9). SERPINE2 binds to its specific target protein to form covalent complexes, which are endocytosed and degraded by cells (10). Through this suicide pathway, SERPINE2 can regulate ECM by inhibiting and

Correspondence to: Professor Danmei Xu, Department of Haematology, Tongji Hospital, Tongji Medical College, Huazhong University of Science and Technology, 1095 Jiefang Avenue, Wuhan, Hubei 430030, P.R. China

E-mail: danmei.xu@imperial.nhs.uk; xudanmei99@yahoo.co.uk

Key words: serine proteinase inhibitor, clade $\mathrm{E}$ member 2 , differential expression, tumor microenvironment, tumor biomarker removing proteases in the local milieu that degrade the ECM by hydrolyzation. As a serine protease inhibitor, SERPINE2 is known to effectively inhibit thrombin, urokinase plasminogen activator (uPA), tissue plasminogen activator (tPA), plasmin, trypsin, XI factor, and prostasin, which is involved in the regulation of blood coagulation and fibrinolysis system $(3,11,12)$.

Increasing evidence has shown that a balance between the extra-cellular serine proteases and their cognate SERPINs plays a key role in cancer progression, thus selected SERPINs might be promising biomarkers for tumor diagnosis or prognosis (13). The potential values of SERPINE2 in the prognosis of several cancers and its co-expression with target proteases in the tumor microenvironment announce its role in tumor progression. SERPINE2 is overexpressed in many cancers including breast cancer (6), pancreatic cancer, gastric cancer, colorectal cancer (14-16), thyroid cancer (17), oral carcinoma (18) and osteosarcoma (19), and contributes to tumor invasion and metastasis. It has been identified that up-regulation of SERPINE2 is related to oncogenic activation of $R A S, B R A F$ and $M E K$, which leads to the pro-neoplastic actions of extracellular signal-regulated kinase $(E R K)$ signaling in intestinal epithelial cells (15). SERPINE2 could also promote drug resistance in osteosarcoma (19) and lymph node metastasis in testicular germ cell tumors (20). Fayard has described a novel pathway where SERPINE2, by binding to lipoprotein receptor-related protein (LRP)-1, stimulates ERK signaling and matrix metalloproteinase (MMP)-9 expressions and promotes the metastatic spread of mammary tumors (6).

In contrast, we previously found that SERPINE2 presents in both normal and benign prostate hypertrophy tissues while its level reduced in prostate carcinoma with a higher Gleason score (21). The SERPINE2-mediated inhibition of uPA serves to prevent prostate cell invasion and metastasis, whereas MMP9 upregulates uPA and facilitates tumor cell invasion through cleavage of SERPINE2 (22). We further showed that SERPINE2 inhibits Hedgehog signaling by reducing the hedgehog ligand Sonic (SHH), which could downregulate downstream molecules, including cyclin D (CCND) 1 and glioma-associated oncogene family zinc finger 1 (GLI1), and as a result inhibits tumor cell proliferation and reduces the expression of B-cell CLL/lymphoma 2 (BCL2) to promote apoptosis (23). SERPINE2 was identified to exert pro-apoptosis activity in prostate carcinoma by down-regulating X-linked 
inhibitor of apoptosis protein (XIAP) through blocking the $\mathrm{NF}-\kappa \mathrm{B}$ signal pathway and changing the stability of XIAP through inhibition of its phosphorylation (8), indicating a protective role of SERPINE2 in tumor progression.

In brain tumors, SERPINE2 plays a more complex role. Pagliara showed that SERPINE2 affects glioma cell migration and invasiveness through the regulation of uPA and MMP-9/2 expression levels, contributing to the degradation of the ECM during tumor invasion (24). However, another study showed that SERPINE2 promoted pre-neoplastic lesion progression to the medulloblastoma owing to aberrant Hedgehog pathway activity independent of the SHH ligand (25).

The biological function of SERPINE2 in different types of tumor appears diverse. In this study, we attempted to determine the expression profiles of SERPINE2 through the EST database, immunohistochemical staining of human tissue microarrays that contains 24 common tumors and normal tissues. The present study sought to systemically review the expression patterns of SERPINE2 in a variety of human normal and tumor tissues, therefore guide us to focus on studying the functional mechanism of SERPINE2 in specific tumors.

\section{Materials and methods}

SERPINE2 transcript data. The SERPINE2 transcript data (UniGene 136630-Hs.38449) were obtained from the EST profile in the NIH UniGene database (http:/www.ncbi.nlm .nih.gov/UniGene/ESTProfileViewer), represented by 1231 ESTs from 282 complementary DNA (cDNA) libraries and corresponding to 4 reference sequences (different isoforms), on January 1st, 2017. The cDNA libraries used for generating the ESTs are themselves derived from a multitude of different organs/tissues/cell types and developmental stages. EST and cDNA sequences provide direct evidence for all of the sampled transcripts, and they are currently the most important resources for transcriptome exploration (26). Data processing was performed using the GraphPad Prism 5 software.

Tissue microarray. Paraformaldehyde-fixed paraffin-embedded human multiple organ tissue microarray slides were purchased from US Biomax Inc. (catalog nos. FDA808c-1 and FDA808c-2; Rockville, MD, USA). The FDA808c-1 slide contained 72 samples of 57 normal tissues and 15 cancer adjacent tissues; the FDA808c- 2 slide contained 13 normal tissues, 5 cancer adjacent tissues and 54 malignant tumor tissues at different pathological stages.

Reagents and antibodies. Rabbit anti-human SERPINE2 monoclonal antibody (Ab) was purchased from Protein Tech Group (Wuhan, China). The EnVision ${ }^{\mathrm{TM}}$ detection kit, including ChemMate ${ }^{\mathrm{TM}}$ EnVision+/HRP, rabbit/mouse and DAB, was purchased from Goodbio Technology (Wuhan, China)

Immunohistochemistry. Immunohistochemical staining was carried out to detect SERPINE2 in situ using an anti-SERPINE2 rabbit anti-human monoclonal antibody according to the manufacturer's protocol. Briefly, the slides were incubated at $60^{\circ} \mathrm{C}$ for $2 \mathrm{~h}$, followed by three 10 -minute cycles of deparaffinization in environmental protection dewaxing liquid and then hydration in a graded ethanol series. The slides were pretreated to promote antigen retrieval in a microwave oven for $15 \mathrm{~min}$ in sodium citrate, $\mathrm{pH}$ 6.0. Endogenous peroxidase in the sections was blocked with hydrogen peroxide $(0.03 \%)$. Bovine serum albumin was added to minimize the background. A 1:50 dilution of the SERPINE2 antibody was applied to the sections, which were then incubated overnight at $4^{\circ} \mathrm{C}$. Next, the sections were incubated with horseradish peroxidase (HRP)-conjugated anti-mouse/rabbit IgG Ab secondary antibody. For visualization, diaminobenzidine (DAB) was used as a chromogen substrate. Both slides were counterstained with hematoxylin. Negative controls were similarly processed by omitting the primary antibody. Tissue microarrays were imaged at 200x and 400x magnification.

\section{Results}

The transcriptional level of SERPINE2 differs among human tissues and organs and changes in the stages of human body growth and development. The EST profile in the NIH UniGene database shows that SERPINE2 is widely expressed in human tissues and organs. We found SERPINE2 transcripts in $77.78 \%$ (35 of 45) of the tested tissues and organs (Fig. 1A). The expression of SERPINE2 varied among different tissues. It was abundant in vascular connective tissue, placenta, skin, and bone tissues, compared to bone marrow, esophagus, ear, embryonic tissue, testis, thyroid, kidney and bladder tissues. No transcript was found in umbilical cord, trachea, tonsil, salivary gland, pituitary gland, pharynx, parathyroid, lymph, ascites and adipose tissues. Beside its variation among different tissues, SERPINE2 expression changed at different stages of development.

It is readily detectable in normal tissues and non-neoplastic tissues, as well as in tumor tissues according to the EST database. Retinoblastoma was the only tumor among the 24 types of tumors without SERPINE2 transcript, indicating that $95.83 \%$ (23 of 24) of the tumor tissues tested expressed SERPINE2 (Fig. 1B). The expression of SERPINE2 differs among different tumors and is significantly higher in skin tumors, chondrosarcoma and germ cell tumors than the other tumor types.

In addition, SERPINE2 is expressed throughout all the stages of human growth, including the embryonic body, blastocyst, fetus, neonate, infant, juvenile and adult stages. As human body grows and develops, SERPINE2 expression begins to climb at the fetus stage, reaches a peak at the neonate and remains steady after entering juvenile period (Fig. 1C).

SERPINE2 is preferentially expressed in gland-rich organs and adenocarcinomas. To investigate the expression profile of SERPINE2, we conducted immunohistochemistry on two sets of tissue microarrays (cat. nos. FDA808c-1 and FDA808c-2, from US Biomax Inc.) which contained various normal tissues and tumor tissues. In consistency with the EST profile, we found that the expression of SERPINE2 was detectable in most of the tissues, especially enriched in glandular organs, such as uterine endometrium, prostate, ovary, colon and digestive organs. The cognate adenocarcinomas abundantly expressed SERPINE2 (Fig. 2A). Skin tumors (basal adenocarcinoma and malignant melanoma of the 
A

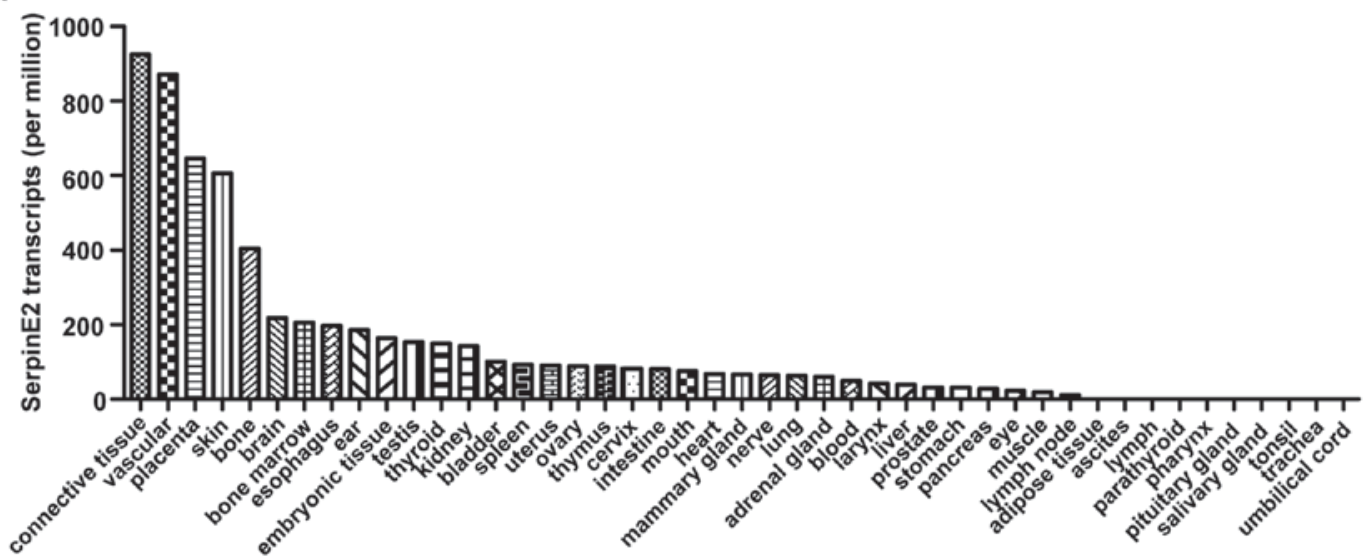

B

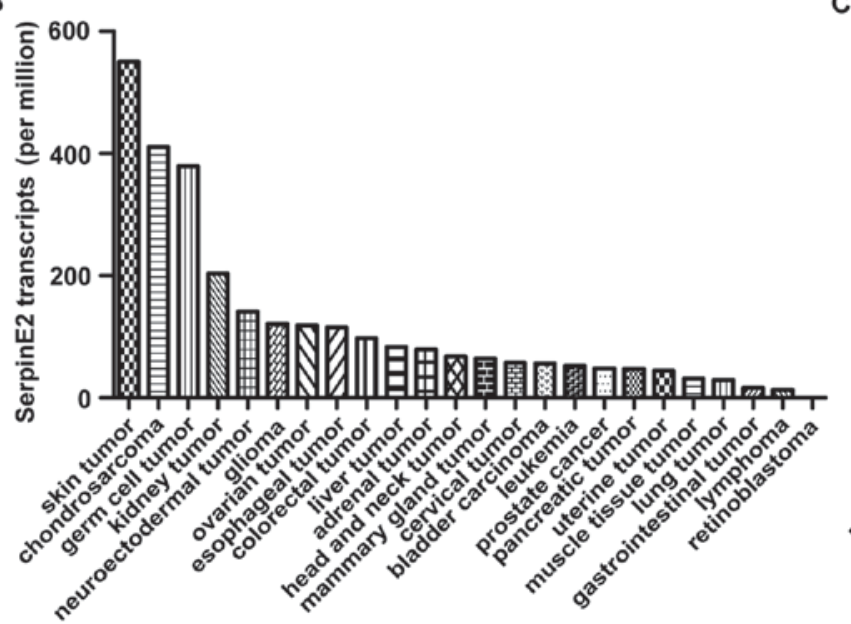

C

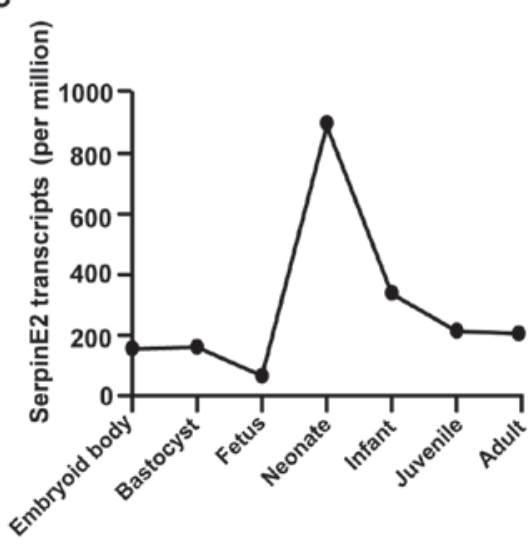

Figure 1. Human SERPINE2 expression patterns in transcription level. Human SERPINE2 transcripts obtained from EST profile in the NIH UniGene database (UniGene 136630-Hs.38449), represented by 1231 ESTs from 282 cDNA libraries, corresponding to 4 reference sequences (different isoforms). (A) SERPINE2 is widely expressed in different tissues and organs as indicated. (B) SERPINE2 is differentially expressed in a variety of tumor tissues as indicated. (C) Human SERPINE2 expression in different developmental stage. SERPINE2, serine proteinase inhibitor, clade E member 2.

skin) as well as liver tumors (hepatocellular carcinoma and hepatoblastoma) exhibited even higher levels of SERPINE2 (Fig. 2B). Notably, the histological expression of SERPINE2 in the skin basically matches its EST level. SERPINE2 was plentiful in the pituitary glands, salivary glands and tonsils (data not shown), in keeping with our previous observation in other gland-rich organs, however not consistent with the EST profile, suggesting post-transcriptional elements may take part in the regulation in these organs. Taken together, SERPINE2 is preferentially expressed in gland-rich organs, especially adenocarcinomas.

SERPINE2 expression differs in most tumors and their counterparts of normal tissues. To understand the differential expression of SERPINE2 in various types of tumors, we compared SERPINE2 expression between tumor and their corresponding normal tissues. For instance, in testis seminoma and lymph nodes with diffuse B cell lymphoma, SERPINE2 expression was remarkably lower than that of their corresponding normal tissues (Fig. 3A). Likewise, SERPINE2 expression was lower in colon adenocarcinoma (Fig. 2A) than that in its normal counterpart. However, breast invasive ductal carcinoma and thyroid papillary carcinoma have significantly higher expression SERPINE2 than their normal counterparts (Fig. 3A). SERPINE2 expression was slightly lower in the benign tumors, such as islet cell tumors and pheochromocytomas of the adrenal gland, in comparison of the corresponding normal tissues (Fig. 3B).

Interestingly, SERPINE2 expressions were more profound in adenocarcinomas of lung and uterine cervix than either normal tissue or the squamous cell carcinomas originated from the same organ (Fig. 3C). The same scenario is also found in skin and uterus adenocarcinoma (data not shown). In summary, SERPINE2 appears highly expressed in the adenocarcinomatous tumor, but with lower levels in squamous cell carcinoma of the same tissue origin, further supporting its preference for glandular tissues.

The role of SERPINE2 was originally highlighted in the brain. Here, we observed high SERPINE2 abundance in the normal cerebrum, normal cerebellum, cerebrum oligodendroglioma and glioblastoma and extremely low levels in atypical meningioma and malignant ependymoma (Fig. 4), all of which are epithelial derived. There are low to medium levels of SERPINE2 expression in mesenchymal tumors such as embryonal rhabdomyosarcoma, osteosarcoma and intestine stromal sarcoma (data not shown). 
A
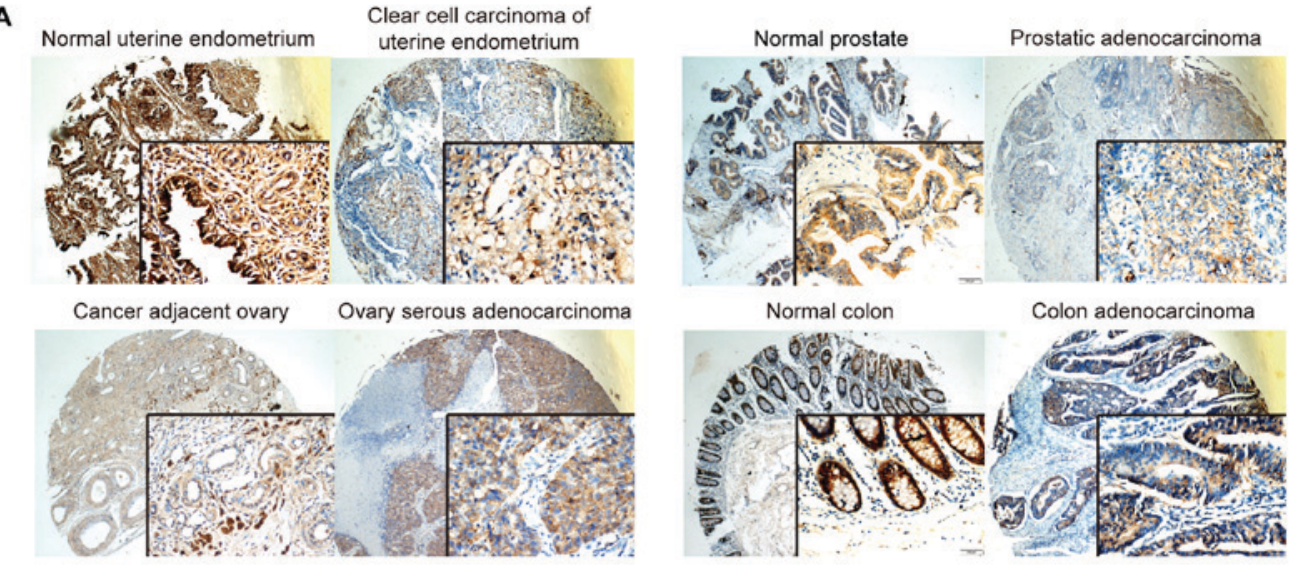

B

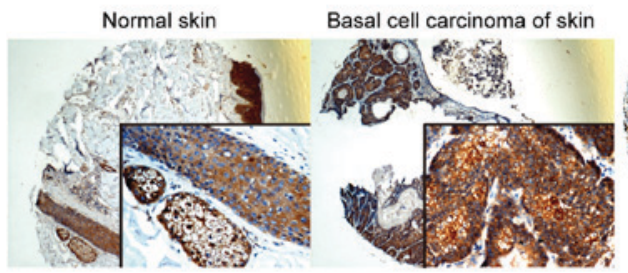

Malignant melanoma of skin

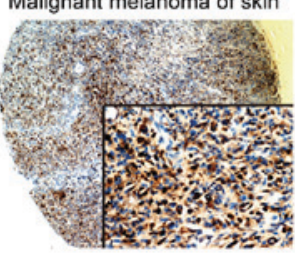

Normal liver

Hepatocellular carcinoma

Hepatoblastoma

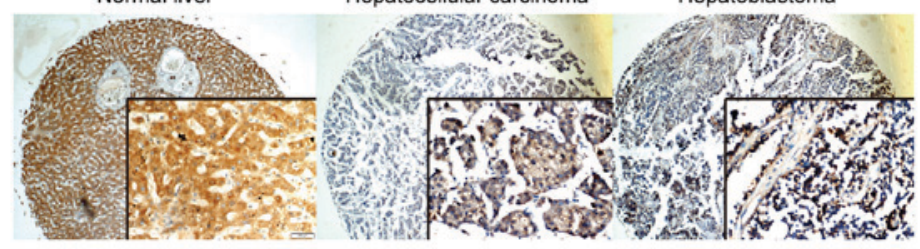

Figure 2. In a number of organs, SERPINE2 protein levels are high in both tumor tissues and their normal counterparts. SERPINE2 protein is stained in brown by immunohistochemistry. (A) SERPINE2 protein expressions were abundant in normal and adenocarcinoma tissues of uterine endometrium, prostate, ovary and colon, which are gland-rich organs. (B) SERPINE2 protein was enriched in normal skin,basal adenocarcinoma and malignant melanoma of skin, as well as in normal liver, hepatocellular carcinoma and hepatoblastoma. Magnification, x200 (main image) and x400 (box at bottom right). SERPINE2, serine proteinase inhibitor, clade E member 2.

The molecular mechanisms how SERPINE2 regulates oncogenesis. Lastly, we provide a diagram to summarize the established molecular mechanism how SERPINE2 regulates oncogenesis (Fig. 5). SERPINE2 inhibits Hedgehog signaling by down-regulating SHH and its down-stream targets including PTCH1, GLI1 and CCND1, thus inhibiting proliferation of prostate cancer cells and conferring protective activity (21). Previously, we found that SERPINE2 has anti-angiogenesis activity in human prostate cancer. Recently, SERPINE2 was found involved in angiogenesis through mediation of midkine and SMAD5 pathways in the retina $(27,28)$.

Moreover, SERPINE2 promotes the apoptosis of prostate cancer cells by reducing the transcriptional level of $X I A P$ through NF- $\mathrm{KB}$ signaling activator $\mathrm{p} 65$ and blunting XIAP activity through Akt signaling. The dual regulation of XIAP can be facilitated through inhibition of uPA (8). In glioma, SERPINE2 knock-down leads to an increase in uPA that indirectly activates MMP9, and enhances glioma cell migration and invasion via the Erk1/2 and p38 pathways through the degradation of ECM components (24). However SERPINE2 was identified to stimulate the Ras-ERK signaling pathway through syndecan-1-mediated internalization in mouse embryonic fibroblasts (29). SERPINE2 was demonstrated to inhibit $M M P 9$ transcriptional activity in SERPINE2 deficient mice (30). By contrast, SERPINE2 was reported to activate MMP9 expression and promote cancer metastasis in mammary tumors (6). Indeed, since the interaction between SERPINE2 and MMP9 is complex, the resultant effects can be discrepant. Furthermore, SERPINE2 was shown to be involved in BRAF- and MEK-induced tumorigenesis in colon epithelial cells and to promote pancreatic cancer invasion by up-regulating ECM production $(14,15)$.

\section{Discussion}

The abundant expression of SERPINE2 in the glandular tissues is prominent in our tissue microarray studies (Figs. 2A and 4A), suggesting its extracellular function may associate with glandular secretion. Indeed, it has been reported that SERPINE2 was highly expressed in the endometrium during the secretory phase compared with the proliferative phase, indicating that it may participate in tissue remodeling during implantation in the placenta and uterus $(31,32)$. A high SERPINE2 level was found in the inhibition of cumulus expansion and oocyte maturation (33). Interestingly, chromogranin A could promote secretory granule biogenesis in endocrine cells through up-regulation of the expression of SERPINE2 via a cAMP-protein kinase A-Sp1 pathway to replenish released granules (34). In consistency with our results, SERPINE2 was found significantly elevated in estrogen receptor (ER) A-negative and in high-grade breast cancer $(6,35)$. ER can dimerize and bind to specific DNA sequences and estrogen 
A
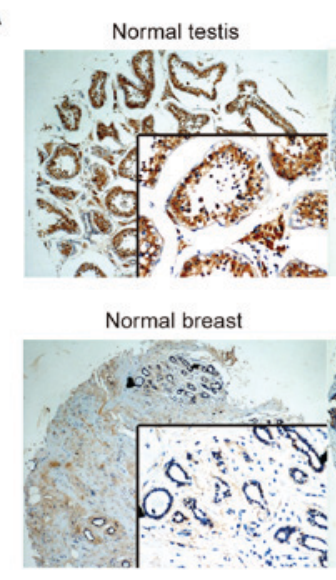

B

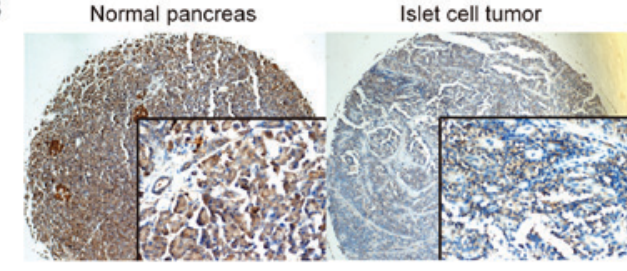

Testis seminoma

Breast invasive ductal carcinoma grade1 stagellA sise 0
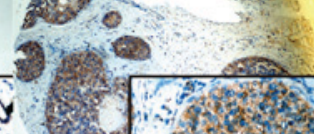

Normal breast
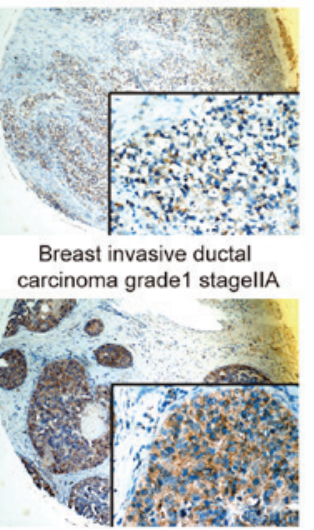

Islet cell tumor

C

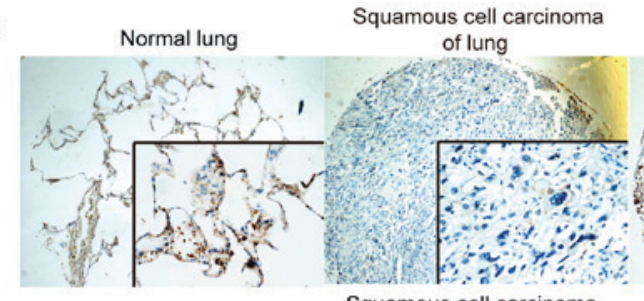

Squamous cell carcinoma

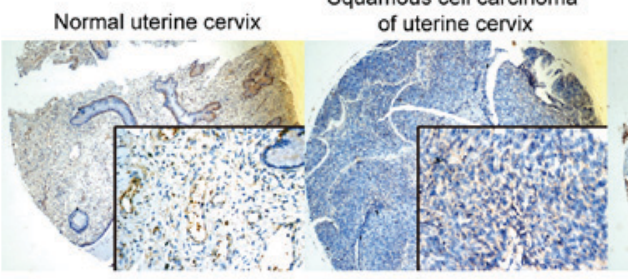

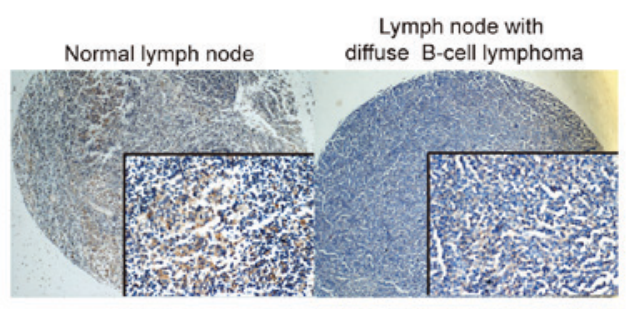
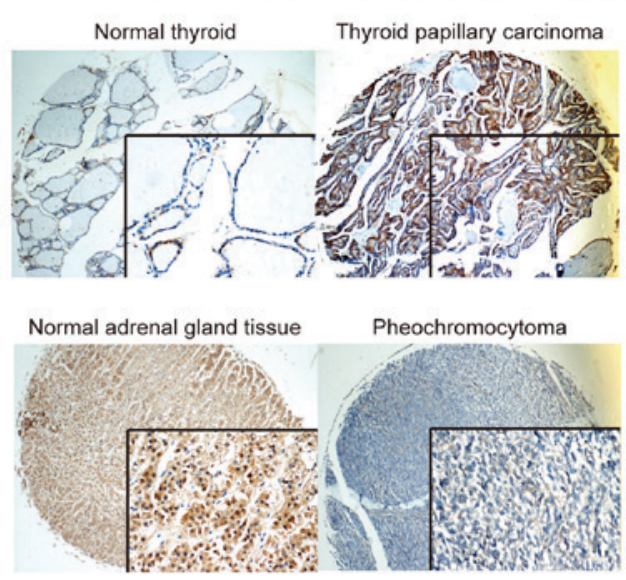

Lung adenocarcinoma

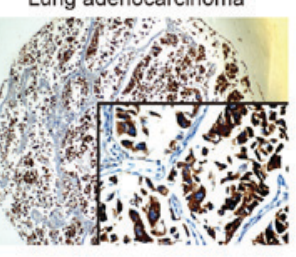

Uterine adenocarcinoma

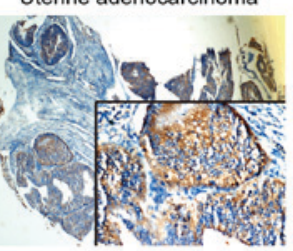

Figure 3. SERPINE2 is expressed differentially in some tumors and their corresponding normal tissues. (A) In malignant tumors, SERPINE2 protein level can be lower (e.g. in testis and lymph node as indicated), or higher (e.g. in breast and thyroid as indicated) than corresponding normal tissues. (B) In benign tumors, SERPINE2 protein level is lower than corresponding normal tissues (e.g. in pancreas and adrenal gland as indicated). (C) SERPINE2 protein level is higher in adenocarcinoma than in their normal counterparts and squamous cell carcinoma of lung and uterine cervix. Magnification, $\mathrm{x} 200$ (main image) and $\mathrm{x} 400$ (box at bottom right). SERPINE2, serine proteinase inhibitor, clade E member 2.

response elements to stimulate estrogen-target gene transcription. It remains unknown whether the regulation of SERPINE2 transcription in breast cancer is ER dependent. Because the involvement of hormones in the progression of many tumors such as estrogen-dependent mammary tumors has been well established, the interaction between hormones and SERPINE2 in tumors may be worthy of further investigation.

Our data show that adenocarcinomatous tissues of the lung and uterine cervix highly express SERPINE2, in contrast to a very scanty expression in squamous cell tumor originated from the same organ (Fig. 3C). In the meanwhile, a low SERPINE2 expression level was found in most mesenchymal tumors (data not show). These findings suggest that SERPINE2 may associate with tumor development of a certain histological subtype. Due to such heterogeneity, the role of SERPINE2 in different subtypes of tumor remains controversial. The protective role of SERPINE2 in tumors was reported in prostate carcinoma and glioma $(8,23,24)$. These studies provide substantial insights into the favorable function of SERPINE2 against tumors by inhibition of tumor growth through the hedgehog pathway and promotion of apoptosis through inhibiting XIAP via the NF- $\kappa$ B pathway and Akt pathway. SERPINE2 can also prevent invasion and metastasis by uPA degradation and lead to reduced activation of the MAPK pathway through down-regulating MMP9. It is also suggested that SERPINE2 may function as an anti-angiogenesis factor by regulating midkine and SMAD5 (28).

On the other hand, Buchholz et al (14), reported that SERPINE2 expression was trivial or absent in all normal pancreas and tissue samples of chronic pancreatitis but abundant in most of the pancreatic carcinoma as well as gastric and colorectal cancer. SERPINE2 overexpression enhances the invasive potential of pancreatic cancer cells 
A

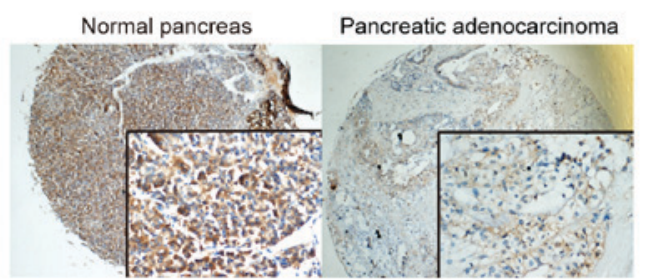

Normal small intestine

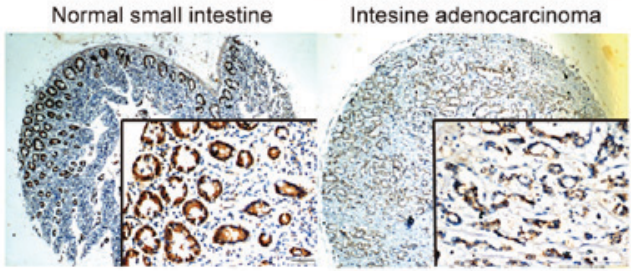

B

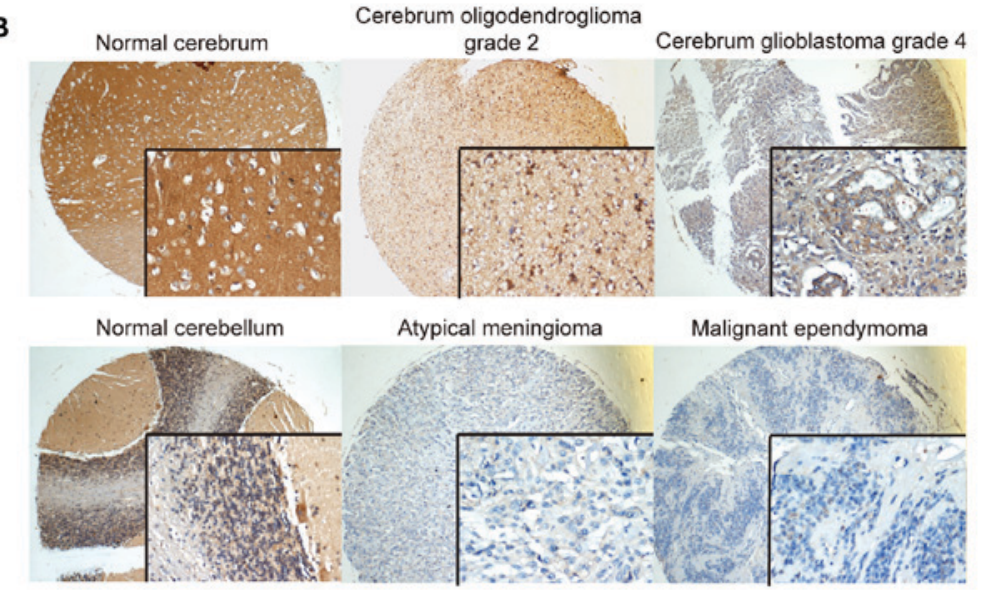

Figure 4. SERPINE2 protein levels in digestive and brain tissues. (A) SERPINE2 protein level is lower in pancreatic adenocarcinoma, signet-ring cell carcinoma of stomach, intestine adenocarcinoma than corresponding normal tissues. (B) SERPINE2 protein is enriched in normal cerebrum, normal cerebellum, cerebrum oligodendroglioma and glioblastoma, and hardly detected in atypical meningioma and malignant ependymoma. Magnification, $\mathrm{x} 200$ (main image) and $\mathrm{x} 400$ (box at bottom right). SERPINE2, serine proteinase inhibitor, clade E member 2.

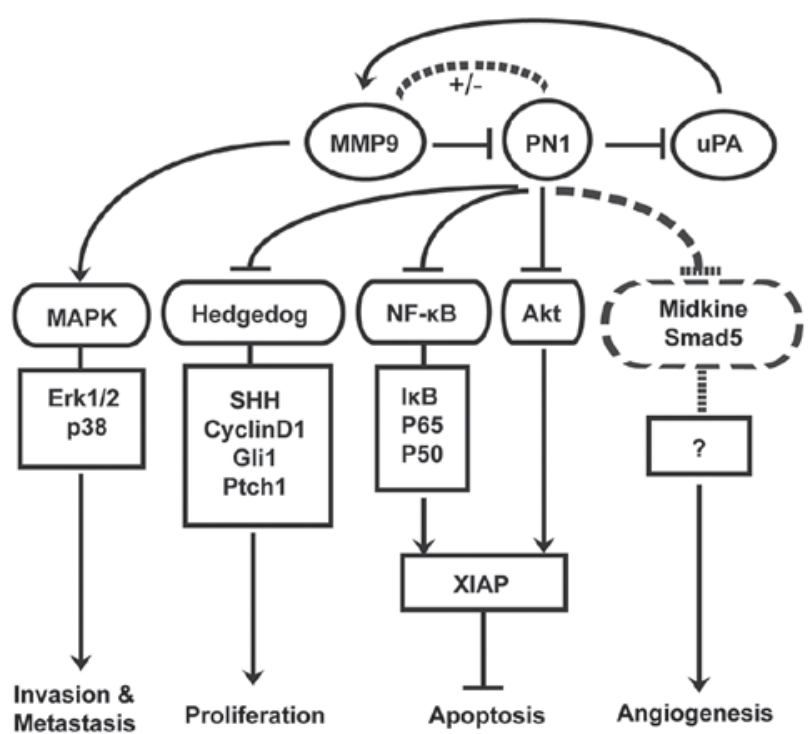

Figure 5. Summary diagram shows how serine proteinase inhibitor, clade E member 2 functions in tumors.

in nude mouse xenografts by altering ECM production and organization within the tumors, thus contributing to the aggressive phenotype of pancreatic cancer (36) and the progression of colorectal cancer by down-regulation of prostasin activity (37). In osteosarcoma, SERPINE2 expression is higher in tissue from patients with metastasis and a tumor-node-metastasis stage II-III, and increases after chemotherapy. In vitro experiments show that SERPINE2 can stimulate cell proliferation and promote drug-resistance in osteosarcoma (19). Thus SERPINE2 appears pro-neoplastic in such scenarios (15). Ultimately, tissue specificity, individual genetic differences, and different expression levels may all contribute to the complicated effects of SERPINE2 in various types of tumors. Therefore, it may be understandable that SERPINE2 can be protective or detrimental in the process of tumor development.

As a glia-derived nexin,SERPINE2 is involved in regulating astrocyte proliferation, neurite outgrowth, neuron migration and localization, contributing to the development of brain, and the regeneration and reconstruction of neurons (38-41). Our studies of EST profile showed that SERPINE2 expression begins to climb at early as the fetus stage, reaches a peak at the neonate and plateaus after the juvenile stage (Fig. 1C), suggesting its roles in human growth and development. The increased expression of SERPINE2 in the neonate stage with the remarkable development of nervous systems annouces its potential role in nervous system development. Indeed, SERPINE2 may contribute to the development of the brain by modulating the proliferation and differentiation of cerebellar granular neuron precursors via the major mitogen $\mathrm{SHH}$, as 
well as via granule neuron migration and positioning (41). SERPINE2 may exert protective activity in the pathalogical progression of intracranial hemorrhage (42), cerebral ischemia (40), and Alzheimer's disease (43). Again, the role of SERPINE2 in tumor progression in the brain seems to be a paradox. The downregulation of SERPINE2 may activate the Erk1/2 and P38 signaling pathways and lead to an increased invasion and metastasis of glioma cells, in which SERPINE2 seems to play a pro-tumor role (24). In another study, however, SERPINE2 may mediate an anti-tumor activity as its deficiency leads to the reduced proliferation of medulloblastoma cells (25). Our tissue microarray shows that SERPINE2 is expressed differentially in different types of brain tumors: abundant in oligodendroglioma and glioblastoma and extremely low in atypical meningioma and malignant ependymoma (Fig. 4).

Our study reveals that expression and function of SERPINE2 may vary in different kinds of tumors. To date, the research on anti-neoplastic mechanisms are more comprehensive than pro-neoplastic ones. The distinctive expression pattern found in this study may help us to understand the function of SERPINE2 in specific tumor types, therefore guide the research of SERPINE2 as a potential tumor marker for diagnosis and prognosis.

\section{Acknowledgements}

The present study was kindly funded by the National Natural Science Foundation of China $(81101572,81372326)$ and the Young Distinguish Talents grant of Tongji Hospital.

\section{References}

1. Barde YA, Lindsay RM, Monard D and Thoenen H: New factor released by cultured glioma cells supporting survival and growth of sensory neurones. Nature 274: 818, 1978 .

2. Guenther J, Nick H and Monard D: A glia-derived neurite-promoting factor with protease inhibitory activity. EMBO J 4: 1963-1966, 1985.

3. Stone SR, Nick H, Hofsteenge J and Monard D: Glial-derived neurite-promoting factor is a slow-binding inhibitor of trypsin, thrombin and urokinase. Arch Biochem Biophys 252: 237-244, 1987.

4. Gloor S, Odink K, Guenther J, Nick H and Monard D: A glia-derived neurite promoting factor with protease inhibitory activity belongs to the protease nexins. Cell 47 : 687-693, 1986.

5. Mansilla S, Boulaftali Y, Venisse L, Arocas V, Meilhac O, Michel JB, Jandrot-Perrus M and Bouton MC: Macrophages and platelets are the major source of protease nexin-1 in human atherosclerotic plaque. Arterioscler Thromb Vasc Biol 28: 1844-1850, 2008.

6. Fayard B, Bianchi F, Dey J, Moreno E, Djaffer S, Hynes NE and Monard D: The serine protease inhibitor protease nexin-1 controls mammarycancermetastasisthroughLRP-1-mediatedMMP-9expression. Cancer Res 69: 5690-5698, 2009.

7. Rosenblatt DE, Cotman CW, Nieto-Sampedro M, Rowe JW and Knauer DJ: Identification of a protease inhibitor produced by astrocytes that is structurally and functionally homologous to human protease nexin-I. Brain Res 415: 40-48, 1987.

8. McKee CM, Ding Y,Zhou J, Li C, Huang L, Xin X, He J, Allen JE, El-Deiry WS, Cao Y, et al: Protease nexin 1 induces apoptosis of prostate tumor cells through inhibition of X-chromosome-linked inhibitor of apoptosis protein. Oncotarget 6: 3784-3796, 2015.

9. Santoro A, Conde J, Scotece M, Abella V, Lois A, Lopez V, Pino J, Gomez R, Gomez-Reino JJ and Gualillo O: SERPINE2 Inhibits IL-1 $\alpha$-Induced MMP-13 Expression in Human Chondrocytes: Involvement of ERK/NF- $\mathrm{B} / \mathrm{AP}-1$ Pathways. PLoS One 10: e0135979, 2015.
10. Crisp RJ, Knauer DJ and Knauer MF: Roles of the heparin and low density lipid receptor-related protein-binding sites of protease nexin 1 (PN1) in urokinase-PN1 complex catabolism. The PN1 heparin-binding site mediates complex retention and degradation but not cell surface binding or internalization. J Biol Chem 275: 19628-19637, 2000.

11. Boulaftali Y, Ho-Tin-Noe B, Pena A, Loyau S, Venisse L, François D, Richard B, Arocas V, Collet JP, Jandrot-Perrus M and Bouton MC: Platelet protease nexin-1, a serpin that strongly influences fibrinolysis and thrombolysis. Circulation 123: 1326-1334, 2011.

12. Kitamura K and Tomita K: Regulation of renal sodium handling through the interaction between serine proteases and serine protease inhibitors. Clin Exp Nephrol 14: 405-410, 2010.

13. Zheng D, Chen H, Davids J, Bryant M and Lucas A: Serpins for diagnosis and therapy in cancer. Cardiovasc Hematol Disord Drug Targets 13: 123-132, 2013.

14. Buchholz M, Biebl A, Neesse A, Wagner M, Iwamura T, Leder G, Adler G and Gress TM: SERPINE2 (protease nexin I) promotes extracellular matrix production and local invasion of pancreatic tumors in vivo. Cancer Res 63: 4945-4951, 2003.

15. Bergeron S, Lemieux E, Durand V, Cagnol S, Carrier JC, Lussier JG, Boucher MJ and Rivard N: The serine protease inhibitor serpinE2 is a novel target of ERK signaling involved in human colorectal tumorigenesis. Mol Cancer 9: $271,2010$.

16. Wang K, Wang B, Xing AY, Xu KS, Li GX and Yu ZH: Prognostic significance of SERPINE2 in gastric cancer and its biological function in SGC7901 cells. J Cancer Res Clin Oncol 141: 805-812, 2015.

17. Stępień T, Brożyna M, Kuzdak K, Motylewska E, Komorowski J, Stępien $H$ and Ławnicka H: Elevated concentrations of SERPINE2/protease nexin-1 and secretory leukocyte protease inhibitor in the serum of patients with papillary thyroid cancer. Dis Markers 2017: 4962137, 2017.

18. Gao S, Krogdahl A, Sørensen JA, Kousted TM, Dabelsteen E and Andreasen PA: Overexpression of protease nexin-1 mRNA and protein in oral squamous cell carcinomas. Oral Oncol 44: 309-313, 2008.

19. Mao M and Wang W: SerpinE2 promotes multiple cell proliferation and drug resistance in osteosarcoma. Mol Med Rep 14: 881-887, 2016.

20. Nagahara A, Nakayama M, Oka D, Tsuchiya M, Kawashima A, Mukai M, Nakai Y, Takayama H, Nishimura K, Jo Y, et al: SERPINE2 is a possible candidate promotor for lymph node metastasis in testicular cancer. Biochem Biophys Res Commun 391: 1641-1646, 2010.

21. McKee CM, Xu D, Cao Y, Kabraji S, Allen D, Kersemans V, Beech J, Smart S, Hamdy F, Ishkanian A, et al: Protease nexin 1 inhibits hedgehog signaling in prostate adenocarcinoma. J Clin Invest 122: 4025-4036, 2012.

22. Xu D, McKee CM, Cao Y, Ding Y, Kessler BM and Muschel RJ: Matrix metalloproteinase-9 regulates tumor cell invasion through cleavage of protease nexin-1. Cancer Res 70: 6988-6998, 2010.

23. McKee CM, Xu D and Muschel RJ: Protease nexin 1: A novel regulator of prostate cancer cell growth and neo-angiogenesis. Oncotarget 4: 1-2, 2013.

24. Pagliara V, Adornetto A, Mammi M, Masullo M, Sarnataro D, Pietropaolo C and Arcone R: Protease Nexin-1 affects the migration and invasion of $\mathrm{C} 6$ glioma cells through the regulation of urokinase plasminogen activator and matrix metalloproteinase-9/2. Biochim Biophys Acta 1843: 2631-2644, 2014.

25. Vaillant C, Valdivieso P, Nuciforo S, Kool M, Schwarzentruber-Schauerte A, Méreau H, Cabuy E, Lobrinus JA, Pfister S, Zuniga A, et al: Serpine2/PN-1 is required for proliferative expansion of pre-neoplastic lesions and malignant progression to medulloblastoma. PLoS One 10: e0124870, 2015.

26. Boguski MS and Schuler GD: ESTablishing a human transcript map. Nat Genet 10: 369-371, 1995.

27. Selbonne S, Azibani F, Iatmanen S, Boulaftali Y, Richard B, Jandrot-Perrus M, Bouton MC and Arocas V: In vitro and in vivo antiangiogenic properties of the serpin protease nexin-1. Mol Cell Biol 32: 1496-1505, 2012.

28. Selbonne S, Francois D, Raoul W, Boulaftali Y, Sennlaub F, Jandrot-Perrus M, Bouton MC and Arocas V: Protease nexin-1 regulates retinal vascular development. Cell Mol Life Sci 72: 3999-4011, 2015. 
29. Li X, Herz J and Monard D: Activation of ERK signaling upon alternative protease nexin-1 internalization mediated by syndecan-1. J Cell Biochem 99: 936-951, 2006.

30. Xu D, Suenaga N, Edelmann MJ, Fridman R, Muschel RJ and Kessler BM: Novel MMP-9 substrates in cancer cells revealed by a label-free quantitative proteomics approach. Mol Cell Proteomics 7: 2215-2228, 2008.

31. Chern SR, Li SH, Lu CH and Chen EI: Spatiotemporal expression of the serine protease inhibitor, SERPINE2, in the mouse placenta and uterus during the estrous cycle, pregnancy, and lactation. Reprod Biol Endocrinol 8: 127, 2010.

32. Lee RK, Fan CC, Hwu YM, Lu CH, Lin MH, Chen YJ and Li SH: SERPINE2, an inhibitor of plasminogen activators, is highly expressed in the human endometrium during the secretory phase. Reprod Biol Endocrinol 9: 38, 2011.

33. Lu CH, Lee RK, Hwu YM, Lin MH, Yeh LY, Chen YJ, Lin SP and $\mathrm{Li} \mathrm{SH}$ : Involvement of the serine protease inhibitor, SERPINE2, and the urokinase plasminogen activator in cumulus expansion and oocyte maturation. PLoS One 8: e74602, 2013.

34. Koshimizu H, Cawley NX, Kim T, Yergey AL and Loh YP: Serpinin: A novel chromogranin A-derived, secreted peptide up-regulates protease nexin-1 expression and granule biogenesis in endocrine cells. Mol Endocrinol 25: 732-744, 2011.

35. Martin KJ, Patrick DR, Bissell MJ and Fournier MV: Prognostic breast cancer signature identified from 3D culture model accurately predicts clinical outcome across independent datasets. PLoS One 3: e2994, 2008.

36. Li Y and Lu YY: Applying a highly specific and reproducible cDNA RDA method to clone garlic up-regulated genes in human gastric cancer cells. World J Gastroenterol 8: 213-216, 2002.
37. Selzer-Plon J, Bornholdt J, Friis S, Bisgaard HC, Lothe IM, Tveit KM, Kure EH, Vogel U and Vogel LK: Expression of prostasin and its inhibitors during colorectal cancer carcinogenesis. BMC Cancer 9: 201, 2009.

38. Cavanaugh KP, Gurwitz D, Cunningham DD and Bradshaw RA: Reciprocal modulation of astrocyte stellation by thrombin and protease nexin-1. J Neurochem 54: 1735-1743, 1990.

39. Lindner J, Guenther J, Nick H, Zinser G, Antonicek H, Schachner M and Monard D: Modulation of granule cell migration by a glia-derived protein. Proc Natl Acad Sci USA 83 4568-4571, 1986.

40. Mirante O,Price M, Puentes W, Castillo X, Benakis C, Thevenet J, Monard D and Hirt L: Endogenous protease nexin-1 protects against cerebral ischemia. Int J Mol Sci 14: 16719-16731, 2013.

41. Vaillant C, Michos O, Orolicki S, Brellier F, Taieb S, Moreno E, Té H, Zeller R and Monard D: Protease nexin 1 and its receptor LRP modulate SHH signalling during cerebellar development. Development 134: 1745-1754, 2007.

42. Wu H, Zhao R, Qi J, Cong Y, Wang D, Liu T, Gu Y, Ban X and Huang Q: The expression and the role of protease nexin-1 on brain edema after intracerebral hemorrhage. J Neurol Sci 270: 172-183, 2008.

43. Vaughan PJ, Su J, Cotman CW and Cunningham DD: Protease nexin-1, a potent thrombin inhibitor, is reduced around cerebral blood vessels in Alzheimer's disease. Brain Res 668: 160-170, 1994. 